\title{
REDUÇÃO NA POPULAÇÃO DE PLANTAS SOBRE A PRODUTIVIDADE E A QUALIDADE FISIOLÓGICA DA SEMENTE DE SOJA ${ }^{1}$
}

\author{
GISELE HERBST VAZQUEZ², NELSON MOREIRA DE CARVALHO ${ }^{3}$, MARIA MADALENA ZOCOLLER BORBA ${ }^{4}$
}

\begin{abstract}
RESUMO - O objetivo deste trabalho foi avaliar os efeitos de reduções na população de plantas sobre a produtividade e a qualidade fisiológica da semente de soja. Duas cultivares de soja (BRSMG 68 Vencedora e M-SOY 8001) foram cultivadas em áreas com e sem o controle de plantas daninhas. As populações estudadas foram: 400.000, 340.000, 280.000, 220.000 e 160.000 pl.ha $^{-1}$, em um espaçamento entrelinhas de $0,43 \mathrm{~m}$. De maneira geral, a presença de plantas daninhas causa decréscimos na produtividade e no tamanho da semente produzida. Entretanto, variações na população não interferem na qualidade fisiológica, no tamanho e na massa de 100 sementes. A cultura da soja é capaz de suportar grandes reduções de população sem perdas significativas de produtividade. Essa capacidade é grandemente influenciável pela cultivar: a Vencedora suporta reduções de até $45 \%$ e a M-SOY 8001 de até 30\%, em relação à população de 400.000 pl.ha ${ }^{-1}$.
\end{abstract}

Termos para indexação: estande, vigor, controle cultural, plantas daninhas, Glycine max

\author{
EFFECTS OF PLANT POPULATION REDUCTIONS ON YIELD AND SEED \\ PHYSIOLOGICAL QUALITY OF SOYBEANS
}

\begin{abstract}
The effects of plant population reductions on soybean plant yield and seed physiological quality were studied. Two soybean cultivars [BRSMG 68 (a.k.a. 'Vencedora') and M-SOY 8001] were grown in areas with and without weed control. The population of 400,000 plants/ hectare was assumed as the referential population and the other treatments consisted of populations of $340,000,280,000,220,000$, and 160,000 plants/hectare, that is, the referential population reduced by $15,30,45$, and $60 \%$, respectively. When applicable, herbicides were used to control weeds. The presence of weeds reduced yield and seed size. Plant population reductions did not interfere in the seed physiological quality, seed size and weight of 100 seeds. Soybean was found to withstand great population reductions without significant reductions in yield. This performance was found to be highly influenced by the genotype: 'Vencedora' withstood up to $45 \%$ and the M-SOY 8001 up to $30 \%$ plant population reductions without significant loss in productivity.
\end{abstract}

Index terms: stand, seed vigor, cultural control, weed control, Glycine max

${ }^{1}$ Submetido em 20/02/2007, Aceito para publicação em 03/10/2007. Parte
da Tese de Doutorado do primeiro autor apresentada a FCAV/UNESP/
Jaboticabal; ${ }^{2}$ Enga. Agra., Dra., Professora, Departamento de Produção
Vegetal, UNICASTELO, CEP 15600-000, Fernandópolis/SP, gisele-agro@, uol.com.br; ${ }^{3}$ Eng. Agr., Dr., Professor Titular, Departamento de Produção Vegetal, FCAV/UNESP, CEP 14884-900, Jaboticabal/SP, nmc@fcav. unesp.br; ${ }^{4}$ Enga. Agra., Dra., Professora Assistente Doutora, Departamento de Economia Rural, FCAV/UNESP, mmzborba@fcav.unesp.br 


\section{INTRODUÇÃO}

Hoje, a população padrão recomendada pela Embrapa

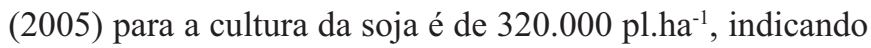
como aceitáveis variações de até $25 \%$ em torno desse valor. Populações muito acima da recomendada, além de acarretar aumentos nos gastos com sementes e um possível acamamento das plantas não proporcionam acréscimos na produtividade. Já a adoção de populações abaixo da recomendada favorece o desenvolvimento de plantas daninhas e pode resultar em plantas muito ramificadas e de altura reduzida, o que também eleva as perdas no momento da colheita.

Na soja, a obtenção de uma lavoura com população adequada depende de diversos fatores, como o bom preparo do solo, a semeadura na época indicada e com disponibilidade hídrica, a utilização correta de herbicidas, a regulagem da semeadora (densidade e profundidade) e a boa qualidade fisiológica da semente empregada. Todavia, freqüentemente, ocorrem reduções na população de plantas em virtude de um desempenho germinativo inadequado de sementes submetidas a condições adversas e que, muitas vezes, não são devidamente aquilatadas pelos agricultores. A não percepção de reduções na população de plantas pelo agricultor ocorre em virtude de que essas variações pouco afetam a produtividade da soja, já que ela é capaz de se adaptar de maneira eficiente aos espaços disponíveis (capacidade de compensação) através de modificações em sua morfologia e nos seus componentes da produtividade (Hicks et al., 1969; Rosolen et al., 1983; Nakagawa et al., 1988 e Denardi et al., 2003).

Quanto ao efeito da população de plantas de soja sobre a qualidade das sementes produzidas, Maeda et al. (1983), trabalhando com três cultivares e três populações de plantas

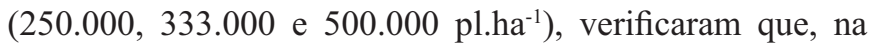
menor população, as sementes apresentaram maior massa de 100 sementes, germinação e vigor. Resultados contrários, entretanto, foram observados por Crusciol et al. (2002), que notaram acréscimos na qualidade fisiológica da semente quando as populações foram elevadas de 300.000 para 500.000 pl.ha $^{-1}$.

Dentro deste contexto, o objetivo deste trabalho foi o de avaliar os efeitos de reduções na população de plantas sobre a produtividade e a qualidade fisiológica das sementes de duas cultivares de soja produzidas em áreas com e sem controle de plantas daninhas.

\section{MATERIAL E MÉTODOS}

O experimento foi conduzido na Universidade Estadual Paulista/UNESP, em Jaboticabal/SP. A instalação ocorreu nos dias 19 e 20/11/2002 e a colheita em 26/03/2003. O solo foi classificado como Latossolo Vermelho eutrófico típico de textura argilosa e, após análise, apresentou as seguintes características químicas: $\mathrm{pH} \mathrm{CaCl}=5,2$; M.O.= $25 \mathrm{~g} \cdot \mathrm{dm}^{-3} ; \mathrm{P}_{\text {resina }}=43 \mathrm{mg} \cdot \mathrm{dm}^{-3} ; \mathrm{K}=4,6 \mathrm{mmol}_{\mathrm{c}} \mathrm{dm}^{-3} ; \mathrm{Ca}+\mathrm{Mg}=$ $50 \mathrm{mmol}_{\mathrm{c}} \mathrm{dm}^{-3}$; CTC $=83,6 \mathrm{mmol}_{\mathrm{c}} \mathrm{dm}^{-3}$ e V= 59\%. Não se efetuou a calagem e a adubação foi calculada segundo Raij et al. (1997) para uma produtividade esperada de 4,0 t.ha- ${ }^{-1}$. Aplicou-se o equivalente a $40 \mathrm{~kg} \mathrm{ha}^{-1} \mathrm{de}_{2} \mathrm{O}_{5}$ e $40 \mathrm{~kg} \cdot \mathrm{ha}^{-1} \mathrm{de}$ $\mathrm{K}_{2} \mathrm{O}$.

O balanço hídrico indicou que não houve deficiências no período de 11/2002 a 04/2003 e sim, excedentes de 75, 252 e $31 \mathrm{~mm}$ em 12/2002, 01/2003 e 02/2003, respectivamente. A temperatura média do período do experimento foi de $24,6^{\circ} \mathrm{C}$ e a umidade relativa de $76,9 \%$.

O delineamento experimental adotado foi o de blocos ao acaso em esquema fatorial ( $5 \times 2)$, compreendendo cinco populações de plantas $(400.000,340.000,280.000,220.000$

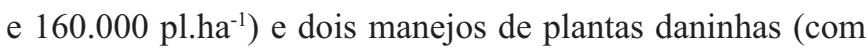
e sem o controle) para as cvs. BRSMG 68 Vencedora e M-SOY 8001 e quatro repetições. Cada parcela foi composta de quatro linhas de $5 \mathrm{~m}$ espaçadas de $0,43 \mathrm{~m}$ e a área útil foi constituída pelas duas fileiras centrais, desprezando-se $0,5 \mathrm{~m}$ das extremidades.

O preparo do solo foi o convencional e, antes da semeadura, as sementes foram tratadas com fungicida (Vitavax-Thiran $200 \mathrm{SC}$ na dose de $250 \mathrm{~mL}$ do produto comercial por $100 \mathrm{~kg}$ de sementes) e, em seguida, inoculadas com Bradyrhizobium spp. O solo foi sulcado e adubado mecanicamente e as sementes foram distribuídas manualmente a uma profundidade $0,05 \mathrm{~m}$ e em quantidade $40 \%$ superior à necessária para estabelecer a densidade de $17,2 \mathrm{pl}^{-1} \mathrm{~m}^{-1}\left(400.000 \mathrm{pl}^{\mathrm{ha}} \mathrm{a}^{-1}\right)$. Nas parcelas conduzidas com controle de plantas daninhas foi efetuada a pulverização manual do herbicida Premerlin 600 CE (3,5 L.ha $\left.{ }^{-1}\right)$, logo após a semeadura. Vinte e quatro dias após a emergência, estando às plantas no estádio $\mathrm{V}_{4}$ de acordo com Fehr e Caviness (1977), todas as parcelas foram desbastadas. Não se procurou eqüidistância entre as plantas, apenas mensurou-se o número inicial de plantas na linha, eliminando-se as que excediam o valor desejado. As populações de 400, 340, 280, 220 e 160.000 pl.ha ${ }^{-1}$ corresponderam a decréscimos no número de sementes germinadas em relação à população de 400.000 pl.ha ${ }^{-1}$ equivalentes a $0,15,30,45$ e $60 \%$. Um mês após a semeadura $\left(\mathrm{V}_{5}\right)$ todas as parcelas com controle de plantas daninhas foram pulverizadas com o herbicida Fusiflex $(1,8$ 
L.ha-1), não havendo necessidade de novas aplicações. O controle de percevejos foi realizado com duas aplicações de Endosulfan $350 \mathrm{CE}$ e duas de Keshet $25 \mathrm{CE}$, nas doses de 1,3 L.ha ${ }^{-1}$ e 0,3 L.ha $^{-1}$, respectivamente. Não ocorreram sintomas de doenças.

No momento da colheita, todas as plantas da parcela útil foram contadas de modo a obter-se a população final e, posteriormente, arrancadas. A porcentagem de sobrevivência (PS) das plantas foi calculada mediante a relação entre a população final e a inicial observada após o desbaste. Conjuntamente, e de maneira aleatória, foram separadas dez plantas por parcela para a determinação da produção por planta (PP), da massa de 100 sementes $\left(\mathrm{M}_{100}\right)$, ambas em g e corrigidas para $13 \%$ de teor de água (b.u.) e da qualidade fisiológica (germinação e vigor) da semente após a trilha manual. $\mathrm{O}$ teste de germinação $(\mathrm{G})$ e a determinação da massa de 100 sementes foram realizados de acordo com as Regras para Análise de Sementes (Brasil, 1992) e os de envelhecimento acelerado (EA), condutividade elétrica (CE) e velocidade de germinação (IVG) segundo Krzyzanowski et al. (1999). As demais plantas foram enfeixadas e encaminhadas para trilha mecanizada. Em seguida, essas sementes foram submetidas a uma desfolha e peneiração e encaminhadas ao laboratório para a avaliação da produtividade (Pr) em kg.ha-1, corrigida para $13 \%$ de teor de água (somando-se com a produção das 10 plantas inicialmente separadas). Todas as sementes trilhadas mecanicamente de cada parcela foram submetidas a uma seqüência de peneiras manuais com crivos circulares $(12,14$, 15,16 e 17), sendo em seguida determinada a porcentagem em peso das sementes retidas em cada uma das peneiras. As sementes trilhadas manualmente foram armazenadas sob condições controladas $\left(10^{\circ} \mathrm{C}\right.$ e $50 \%$ de UR do ar) e, após cinco meses, efetuaram-se novas determinações da qualidade fisiológica.

A análise estatística dos dados foi realizada separadamente para cada uma das cultivares utilizandose o programa ESTAT 2.0 (1994). Os resultados do fator quantitativo (população), quando significativos pelo teste $\mathrm{F}$, foram analisados pelo teste de Tukey a $5 \%$ de probabilidade e por regressão polinomial e os do qualitativo (manejo), apenas por Tukey.

\section{RESULTADOS E DISCUSSÃO}

$\mathrm{O}$ manejo de plantas daninhas interferiu sobre a $\mathrm{Pr}$ das duas cultivares analisadas, sendo estas superiores nas áreas com herbicidas (Tabela 1). Reduções na Pr da soja ocasionadas pela competição com plantas daninhas já eram esperadas. Neste experimento, o decréscimo atribuído a mato interferência foi de $12,8 \%$ para a cv. Vencedora e de 10,6\% para a M-SOY, valores estes baixos, em comparação aos apresentados por Blanco et al. (1973), que podem chegar a $90 \%$. O baixo valor poderia ser explicado pelo fato de que o local utilizado vinha sendo há tempo cultivado e pulverizado com herbicidas, o que deve ter reduzido a pressão das infestantes, além das boas condições pluviométricas, que em muito favoreceram a produtividade. Em relação à $\mathrm{PP}, \mathrm{a} \mathrm{cv}$. M-SOY cultivada em áreas sem controle de plantas daninhas apresentou uma redução de $11,8 \%$ quando comparada às que cresceram em áreas com controle com herbicidas.

O fator população interferiu em todos os parâmetros analisados (Tabela 1). Não houve diferenças significativas quanto a PS das plantas em função da população da cv. Vencedora, apesar do teste $\mathrm{F}$ ter mostrado efeito significativo. Já na cv. M-SOY, a PS da população de 160.000 pl.ha-1 foi inferior estatisticamente à da de 220.000 pl.ha $^{-1}$. A PS das plantas foi, em média, de $89 \%$ na Vencedora e de $90 \%$, na M-SOY.

Decréscimos na população de até $45 \%$, na cv. Vencedora, e de até $30 \%$ na da M-SOY, não causaram reduções significativas na $\operatorname{Pr}$ (Tabela 1). Estes resultados estão de acordo com os relatados por Rosolem et al. (1983), Nakagawa et al. (1988) e Denardi et al. (2003), os quais mostraram que a soja suporta ampla variação no número de plantas por área, sem afetar significativamente a produtividade, graças à grande capacidade de ajuste de seus componentes de produção. Segundo a Embrapa (2005), populações de 240.000 a 400.000 pl.ha $^{-1}$ são aceitáveis em função da cultivar, regime de chuvas, data de semeadura e fertilidade do solo. Portanto, os resultados apresentados pela M-SOY estiveram dentro destes limites; já a Vencedora suportou decréscimos para valores de até 220.000 pl.ha-1.

As equações deregressãoe os coeficientes dedeterminação obtidos nas análises de regressão para a produtividade em função da população para as duas cultivares, estão apresentados na Figura 1 e indicam que se podem esperar reduções de 151 e de 136 kg.ha-1 para as cvs. Vencedora e M-SOY, respectivamente, a cada redução de 15\% (60.000 pl.ha-1) na população de plantas. Nas diversas populações estudadas, a cv. Vencedora apresentou, em termos médios, uma produtividade $883 \mathrm{~kg}$ superior à da M-SOY. 
TABELA 1. Análise da variância (quadrado médio) e médias da população de plantas na colheita, da porcentagem desobrevivência de plantas na colheita (PS), da produção por planta (PP) e da produtividade (Pr) em função do manejo das plantas daninhas e da população de plantas de soja das cultivares BRSMG 68 Vencedora (A) e M-SOY 8001 (B) ${ }^{1}$, Jaboticabal, SP, 2002/03.

\begin{tabular}{|c|c|c|c|c|}
\hline \multicolumn{5}{|c|}{ (A) BRSMG 68 VENCEDORA } \\
\hline Fatores & $\begin{array}{c}\text { Populaçãa } \\
\left(\text { pl.ha }^{-1}\right)\end{array}$ & $\begin{array}{l}P^{2} \\
(\%)\end{array}$ & $\begin{array}{c}\text { PP } \\
\left(\text { g.pl }^{-1}\right)\end{array}$ & $\begin{array}{c}\text { Pr } \\
\left.\text { (kg.ha }^{-1}\right)\end{array}$ \\
\hline Manejo (M) & $865,75 \times 10^{6^{*}}$ & $46,50 \mathrm{~ns}$ & $72,48 * *$ & $4109059,9^{* *}$ \\
\hline População (P) & $48503,6 \times 10^{6 * *}$ & $82,41 *$ & $365,45 * *$ & $643310,81 *$ \\
\hline $\mathrm{MxP}$ & $445,3 \times 10^{6} \mathrm{~ns}$ & $26,70 \mathrm{~ns}$ & $20,41 *$ & $197311,9 \mathrm{~ns}$ \\
\hline Média geral & 247.385 & (89) 71,27 & 20,56 & 4.679 .2 \\
\hline \multicolumn{5}{|l|}{ Manejo $\mathrm{Pl}$. daninha } \\
\hline Com Herbicida - CH & $252.037 \mathrm{~A}$ & (90) 72,35 & - & $4.999,7 \mathrm{~A}$ \\
\hline Sem Controle - SC & $242.732 \mathrm{~B}$ & (87) 70,19 & - & $4.358,7 \mathrm{~B}$ \\
\hline DMS & 8.772 & 3,53 & Manejo & 294,4 \\
\hline População & & & $\mathrm{CH} \quad \mathrm{SC}$ & \\
\hline 400.000 & $337.209 \mathrm{~A}$ & (84) 66,78 & $15,4 \mathrm{BCa}$ & $5.139,6 \mathrm{~A}$ \\
\hline 340.000 & $301.241 \mathrm{~B}$ & (89) 70,45 & $14,6 \mathrm{Ca}$ & $4.589,1 \mathrm{AB}$ \\
\hline 280.000 & $254.360 \mathrm{C}$ & (91) 74,64 & $18,4 \mathrm{BCa}$ & $4.747,6 \mathrm{AB}$ \\
\hline 220.000 & $202.761 \mathrm{D}$ & (92) 74,12 & $19,9 \mathrm{Bb}$ & $4.478,7 \mathrm{AB}$ \\
\hline 160.000 & $141.352 \mathrm{E}$ & (88) 70,34 & $35,3 \mathrm{Aa}$ & $4.441,2 \mathrm{~B}$ \\
\hline DMS & 19.735 & 7,95 & coluna 5,3 linha 3,8 & 662,3 \\
\hline $\mathrm{CV}(\%)$ & 5,46 & 7,64 & 12,59 & 9,69 \\
\hline \multicolumn{5}{|l|}{ (B) M-SOY 8001} \\
\hline Manejo (M) & $5,52 \times 10^{6} \mathrm{~ns}$ & $0,47 \mathrm{~ns}$ & $43,71 * *$ & $1816221,5^{* *}$ \\
\hline População (P) & $56532,3 \times 10^{6 * *}$ & $113,88 *$ & $265,76^{* *}$ & $544178,0 * *$ \\
\hline $\mathrm{M} \times \mathrm{P}$ & $436,9 \times 10^{6} \mathrm{~ns}$ & $43,47 \mathrm{~ns}$ & $10,06 \mathrm{~ns}$ & $100233,6 \mathrm{~ns}$ \\
\hline Média geral & 251.227 & (90) 72,31 & 16,6 & $3.796,0$ \\
\hline \multicolumn{5}{|l|}{ Manejo PI. daninha } \\
\hline Com Herbicida - CH & 250.856 & (90) 72,20 & $17,7 \mathrm{~A}$ & $4.009,1 \mathrm{~A}$ \\
\hline Sem Controle - SC & 251.599 & (90) 72,41 & $15,6 \mathrm{~B}$ & $3.582,9 \mathrm{~B}$ \\
\hline DMS & 11.651 & 3,77 & 1,44 & 234,9 \\
\hline \multicolumn{5}{|l|}{ População } \\
\hline 400.000 & $356.468 \mathrm{~A}$ & (89) $71,57 \mathrm{AB}$ & $11,7 \mathrm{C}$ & $4.162,1 \mathrm{~A}$ \\
\hline 340.000 & $304,142 \mathrm{~B}$ & (89) $71,88 \mathrm{AB}$ & $12,4 \mathrm{C}$ & $3.781,8 \mathrm{AB}$ \\
\hline 280.000 & $246.366 \mathrm{C}$ & (88) $70,77 \mathrm{AB}$ & $16,1 \mathrm{~B}$ & $3.920,2 \mathrm{AB}$ \\
\hline 220.000 & $210.755 \mathrm{D}$ & (96) $78,67 \mathrm{~A}$ & $16,7 \mathrm{~B}$ & $3.490,4 \mathrm{~B}$ \\
\hline 160.000 & $138.404 \mathrm{E}$ & (86) $68,65 \mathrm{~B}$ & $26,2 \mathrm{~A}$ & $3.625,6 \mathrm{~B}$ \\
\hline DMS & 26.212 & 8,47 & 3,24 & 528,4 \\
\hline $\mathrm{CV}(\%)$ & 7,14 & $\frac{8.02}{c 102 \sin }$ & $\frac{13.35}{13.35}$ & 9.53 \\
\hline
\end{tabular}


FIGURA 1. Relação entre produção por planta (PP) e produtividade (Pr) com as diferentes populações estudadas das cvs. BRSMG 68 Vencedora e M-SOY $8001 \mathrm{em}$ áreas com e sem controle de plantas daninhas.

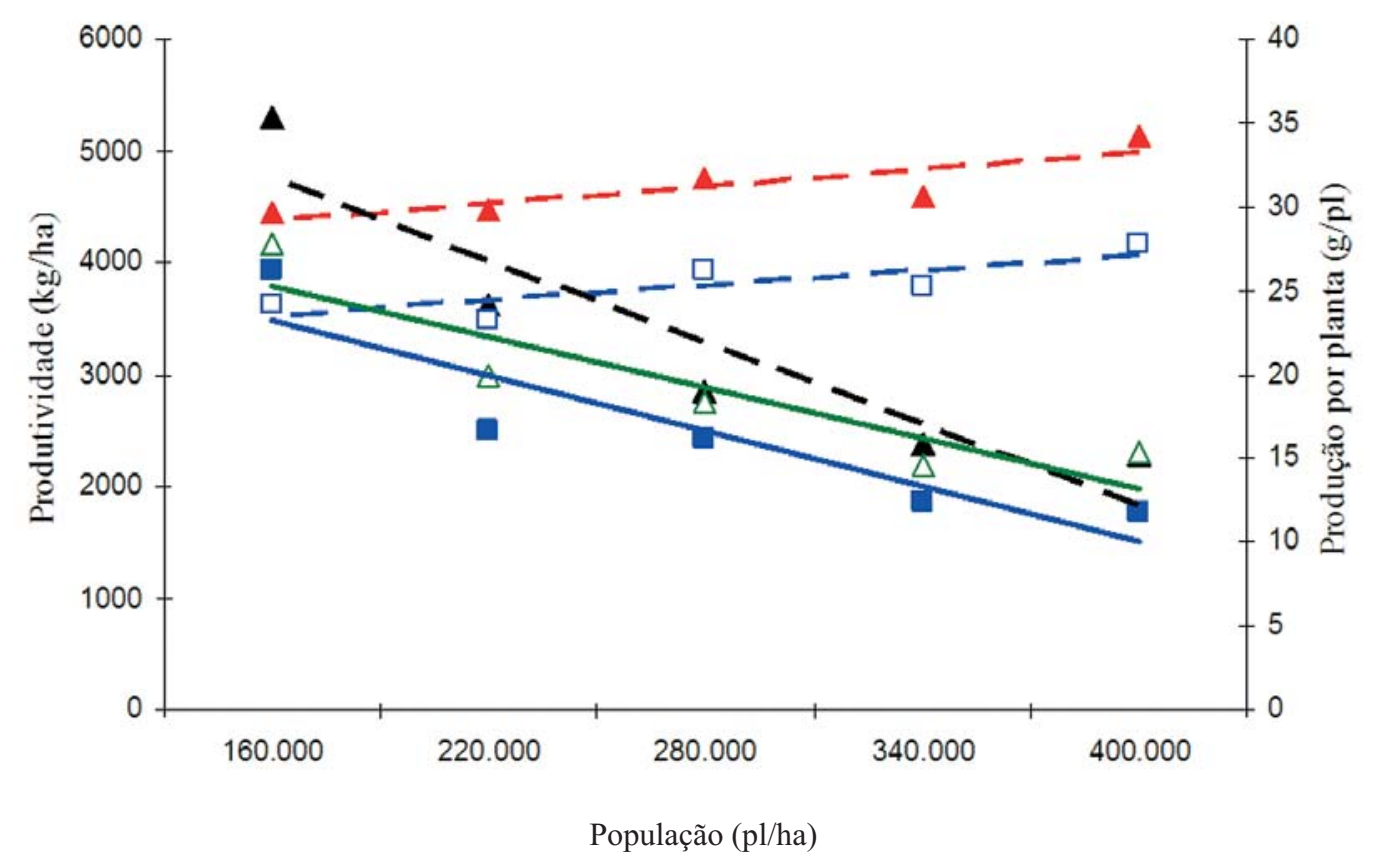

$\mathrm{PP}($ Venc $\mathrm{CH})=-0,000081 \mathrm{x}+44,593 \mathrm{R}^{2}=0,86$

$\mathrm{PP}($ Venc $\mathrm{SC})=-0,00005 \mathrm{x}+33,217 \mathrm{R}^{2}=0,82$

$\mathrm{PP}(\mathrm{M}-\mathrm{SOY})=-0,0000551 \mathrm{x}+32,068 \mathrm{R}^{2}=0,82$

$\operatorname{Pr}($ Venc $)=0,002512 \mathrm{x}+3976 \mathrm{R}^{2}=0,71$

$\operatorname{Pr}(\mathrm{M}-\mathrm{SOY})=0,002274 \mathrm{x}+3159,3 \mathrm{R}^{2}=0,61$

$\mathrm{CH}$ - com aplicação de herbicidas e $\mathrm{SC}$ - sem controle de plantas daninhas $5 \%$ de probabilidade. ${ }^{(2)}$ Médias transformadas em arcsen $\sqrt{ } \mathrm{x} / 100$ e médias originais entre parênteses. ${ }^{*} \mathrm{e} * *$ significativo a $5 \%$ e a $1 \%$ de probabilidade, respectivamente, pelo teste $\mathrm{F}$, ns - não significativo.

Quanto à PP, ocorreu um aumento significativo com a redução da população da cv. M-SOY (Tabela 1B), o que está de acordo com o relatado por Pereira (1989). Esses dados evidenciam a grande capacidade de compensação da soja, pois que, da população de 400.000 para a de 160.000 pl.ha ${ }^{-1}$, houve uma redução na produtividade de apenas $13 \%$, pequena redução esta que foi conseqüência de um aumento aproximado de $123 \%$ na capacidade de PP.

Houve interação dos fatores $\mathrm{M}$ e P quanto à PP da cv. Vencedora (Tabela 1A). Analisando as diferentes populações, no menor valor $\left(160.000 \mathrm{pl}^{-h^{-1}}\right)$, tanto em áreas com controle como nas sem controle de plantas daninhas, os valores obtidos superaram estatisticamente aos demais. Apenas nas menores populações (160.000 e 220.000 pl.ha $^{-1}$ ), o manejo de daninhas resultou em maior PP, quando comparadas às áreas sem controle. Com reduções de até $30 \%$ na população, a cv. Vencedora foi capaz de competir eficientemente com as plantas daninhas (controle cultural), pois não houve diferenças estatísticas entre as médias dos dois tratamentos de manejo (CH e SC). Para reduções maiores que $30 \%$, cada planta de soja, em áreas sem controle, sofreu uma redução média de $19,4 \%$ na sua capacidade de produção em relação às áreas com controle.

As equações deregressãoe os coeficientes de determinação resultantes das análises de regressão da PP em função da população das duas cultivares estão apresentados na Figura 1 e mostram que se poderiam esperar aumentos de 3,3 g.pl-1 da cv. M-SOY e de 3,0 e 4,9 g.pl $\mathrm{l}^{-1}$ da cv. Vencedora em áreas sem e com o controle de plantas daninhas, respectivamente, para cada decréscimo de $15 \%$ (60.000 pl.ha-1) na população de plantas.

O resumo da análise de variância e as médias dos valores de G, EA, CE, IVE avaliados após a colheita (1 ${ }^{\mathrm{a}}$ época) e 5 meses após ( $2^{a}$ época), além da massa de 100 sementes, encontram-se nas Tabelas 2 e 3 . O manejo de plantas daninhas não interferiu na germinação das sementes das 
duas cultivares, que apresentaram elevado valor mesmo após 5 meses de armazenamento (Tabela 2). Quanto ao vigor avaliado através dos testes de EA e de CE, ambos após o armazenamento ( $2^{\mathrm{a}}$ época), as sementes da cv. M-SOY
(Tabela 2B) provenientes das áreas sem controle exibiram desempenho estatisticamente superior ao das de áreas com controle. As sementes da Vencedora não mostraram diferenças.

TABELA 2. Análise de variância (quadrado médio) e médias da porcentagem de germinação (G), do envelhecimento acelerado (EA) e da condutividade elétrica (CE) em duas épocas ( $\mathrm{l}^{\mathrm{a}}$ época - logo após a colheita e $2^{\mathrm{a}}$ época - 5 meses após) em função do manejo das plantas daninhas e da população de plantas de soja das cultivares BRSMG 68 Vencedora (A) e M-SOY 8001 (B) ${ }^{1}$, Jaboticabal, SP, 2002/03.

\begin{tabular}{|c|c|c|c|c|c|c|c|}
\hline \multicolumn{8}{|c|}{ (A) BRSMG 68 VENCEDORA } \\
\hline \multirow{2}{*}{ Fatores } & \multicolumn{2}{|c|}{${ }^{(2)} \mathrm{G}(\%)$} & \multicolumn{2}{|c|}{ (2) $\mathrm{EA}(\%)$} & \multicolumn{3}{|c|}{$\mathrm{CE}\left(\mu \mathrm{S} \cdot \mathrm{cm}^{-1} \cdot \mathrm{g}^{-1}\right)$} \\
\hline & $1^{\mathrm{a}}$ época & $2^{\mathrm{a}}$ época & $1^{\mathrm{a}}$ época & $2^{\mathrm{a}}$ época & 1 ápoca & \multicolumn{2}{|c|}{ 2a época } \\
\hline Manejo-M & $19.06 \mathrm{~ns}$ & $15,74 \mathrm{~ns}$ & $0,21 \mathrm{~ns}$ & $1,37 \mathrm{~ns}$ & $9,11 \mathrm{~ns}$ & \multicolumn{2}{|c|}{$170,69 * *$} \\
\hline População- P & $80,44 n s$ & $38,01 \mathrm{~ns}$ & $18,73 \mathrm{~ns}$ & $28,10 \mathrm{~ns}$ & $0,88 \mathrm{~ns}$ & \multicolumn{2}{|c|}{$29,93 \mathrm{~ns}$} \\
\hline $\mathrm{M} \times \mathrm{P}$ & $19,44 \mathrm{~ns}$ & $21,61 \mathrm{~ns}$ & $7,81 \mathrm{~ns}$ & $24,85 \mathrm{~ns}$ & $12,21 \mathrm{~ns}$ & \multicolumn{2}{|c|}{$90,92 * *$} \\
\hline Média geral & (97) 82,2 & (98) 83,4 & (93) 74,8 & $(86) 68,3$ & 61,81 & \multicolumn{2}{|c|}{72,44} \\
\hline \multicolumn{8}{|c|}{ Manejo Pl. daninha } \\
\hline $\mathrm{CH}$ & $(97) 81,5$ & (97)82.7 & (93) 74,7 & $(86) 68,5$ & 62,28 & \multicolumn{2}{|c|}{ - } \\
\hline $\mathrm{SC}$ & (97) 83,0 & $(98) 84,0$ & (93) 74,8 & (86) 68,1 & 61,33 & \multicolumn{2}{|c|}{ - } \\
\hline DMS & 3,9 & 3,59 & 2,03 & 2,25 & 2,0 & \multicolumn{2}{|c|}{ Manejo } \\
\hline População & & & & & & $\mathrm{CH}$ & $\mathrm{SC}$ \\
\hline 400.000 & $(95) 77,9$ & $(99) 85,8$ & $(91) 72,6$ & $(84) 66,5$ & 61,7 & $71,16 \mathrm{Aa}$ & $70,49 \mathrm{ABa}$ \\
\hline 340.000 & (99) 85,9 & $(97) 82,2$ & $(94) 75,9$ & $(90) 71,4$ & 62,17 & $73,03 \mathrm{Aa}$ & $74,29 \mathrm{Ba}$ \\
\hline 280.000 & (97)82,1 & (99) 85,3 & $(92) 74,0$ & $(85) 67,1$ & 62,14 & $75,74 \mathrm{ABb}$ & $73,59 \mathrm{Ba}$ \\
\hline 220.000 & (96) 80,7 & (97) 80,6 & (93) 75,1 & $(86) 68,1$ & 61,56 & $80,88 \mathrm{Ba}$ & $65,08 \mathrm{Aa}$ \\
\hline 160.000 & (98) 84,5 & (98) 83,0 & (94) 76,3 & $(86) 68,3$ & 61,46 & $71,71 \mathrm{Aa}$ & $68,42 \mathrm{ABa}$ \\
\hline DMS & 8.7 & 8,1 & 4,6 & 5,1 & 4,5 & coluna 7. & inha 5,23 \\
\hline $\mathrm{CV}(\%)$ & 7,29 & 6,63 & 4,18 & 5,08 & 4,98 & & \\
\hline \multicolumn{8}{|c|}{ (B) M-SOY 8001} \\
\hline Manejo- M & $17,61 \mathrm{~ns}$ & $2,21 \mathrm{~ns}$ & $14,30 \mathrm{~ns}$ & $383,93 * *$ & $11,60 \mathrm{~ns}$ & \multicolumn{2}{|c|}{$128,63 * *$} \\
\hline População- P & $9,47 \mathrm{~ns}$ & $10,69 \mathrm{~ns}$ & $7,96 \mathrm{~ns}$ & $32,68 \mathrm{~ns}$ & $17,42 \mathrm{~ns}$ & \multicolumn{2}{|c|}{$17,29 \mathrm{~ns}$} \\
\hline $\mathrm{MxP}$ & $36,37 \mathrm{~ns}$ & $36,30 \mathrm{~ns}$ & $9,25 \mathrm{~ns}$ & $42.97 \mathrm{~ns}$ & $8,15 \mathrm{~ns}$ & \multicolumn{2}{|c|}{$5.59 \mathrm{~ns}$} \\
\hline Média geral & $(96) 80.2$ & $(98) 82,6$ & $(95) 77,5$ & (93) 76,0 & 67,91 & \multicolumn{2}{|c|}{77,56} \\
\hline \multicolumn{8}{|c|}{ Manejo Pl. daninha } \\
\hline $\mathrm{CH}$ & (97) 80,9 & $(98) 82,3$ & (95) 78,1 & (91) $72.9 \mathrm{~B}$ & 68,44 & \multicolumn{2}{|c|}{$79.36 \mathrm{~B}$} \\
\hline $\mathrm{SC}$ & (96)79.6 & (98) 82,8 & (94)76.9 & (95) $79,1 \mathrm{~A}$ & 67,37 & \multicolumn{2}{|c|}{$75.77 \mathrm{~A}$} \\
\hline DMS & 3,7 & 3,4 & 2,1 & 3,1 & 1,87 & \multicolumn{2}{|c|}{2,97} \\
\hline \multicolumn{8}{|l|}{ População } \\
\hline 400.000 & $(96) 80,6$ & (97)80,8 & (94) 76,8 & (93) 76.2 & 67,66 & \multicolumn{2}{|c|}{76,51} \\
\hline 340.000 & $(96) 79,4$ & $(98) 83,8$ & $(95) 77,6$ & (94) 78,2 & 66,1 & \multicolumn{2}{|c|}{79,84} \\
\hline 280.000 & $(96) 79,7$ & $(97) 82,3$ & $(94) 76,5$ & (95) 77,1 & 67,17 & \multicolumn{2}{|c|}{76,1} \\
\hline 220.000 & $(98) 82,0$ & $(98) 82,6$ & $(95) 77,4$ & (93) 75,5 & 69,99 & & \\
\hline 160.000 & $(95) 79,4$ & $(98) 83,4$ & $(96) 79,1$ & (90) 72,8 & 68,61 & & \\
\hline DMS & 8,4 & 7,7 & 4,7 & 6,9 & 4,2 & & \\
\hline $\mathrm{CV}(\%)$ & 7,16 & 6,36 & 4,19 & 6,21 & 4,24 & & \\
\hline
\end{tabular}

(Médias seguidas pelas mesmas letras, maiúsculas na coluna e minúsculas nas linhas, não diferem estatisticamente entre si pelo teste de Tukey a $5 \%$ de probabilidade. (2)Médias transformadas em arcsen $\bigvee_{\mathrm{X}} / 100-$ médias originais entre parênteses. ${ }^{*} \mathrm{e} * *$ significativo a $5 \%$ e a $1 \%$ de probabilidade, respectivamente, ns - não significativo. $\mathrm{CH}$ - com herbicida e $\mathrm{SC}$ - sem controle 
A variação da população não provocou alterações na porcentagem de $\mathrm{G}$ e de EA das duas cultivares (Tabela 2). Rezende et al. (1985) e Moore (1991) também não verificaram influência significativa da população sobre a germinação e o vigor de sementes de soja.

Os resultados da interação dos fatores $\mathrm{M}$ e $\mathrm{P}$ sobre os dados de CE ( $2^{\mathrm{a}}$ época - Tabela 2A), IVE (1 ${ }^{\mathrm{a}}$ e $2^{\mathrm{a}}$ épocas - Tabela 3A) da cv. Vencedora e de IVE (1 ${ }^{\text {a }}$ época Tabela 3B) da cv. M-SOY não obstante terem se mostrado estatisticamente significativos, não indicaram uma tendência lógica de resultados.

TABELA 3. Análise de variância (quadrado médio) e médias do índice de velocidade de emergência (IVE) e da massa de 100 sementes $\left(M_{100}\right)$ em função do manejo das plantas daninhas e da população de plantas de soja das cultivares BRSMG 68 Vencedora (A) e M-SOY 8001 (B)1, Jaboticabal, SP, 2002/03.

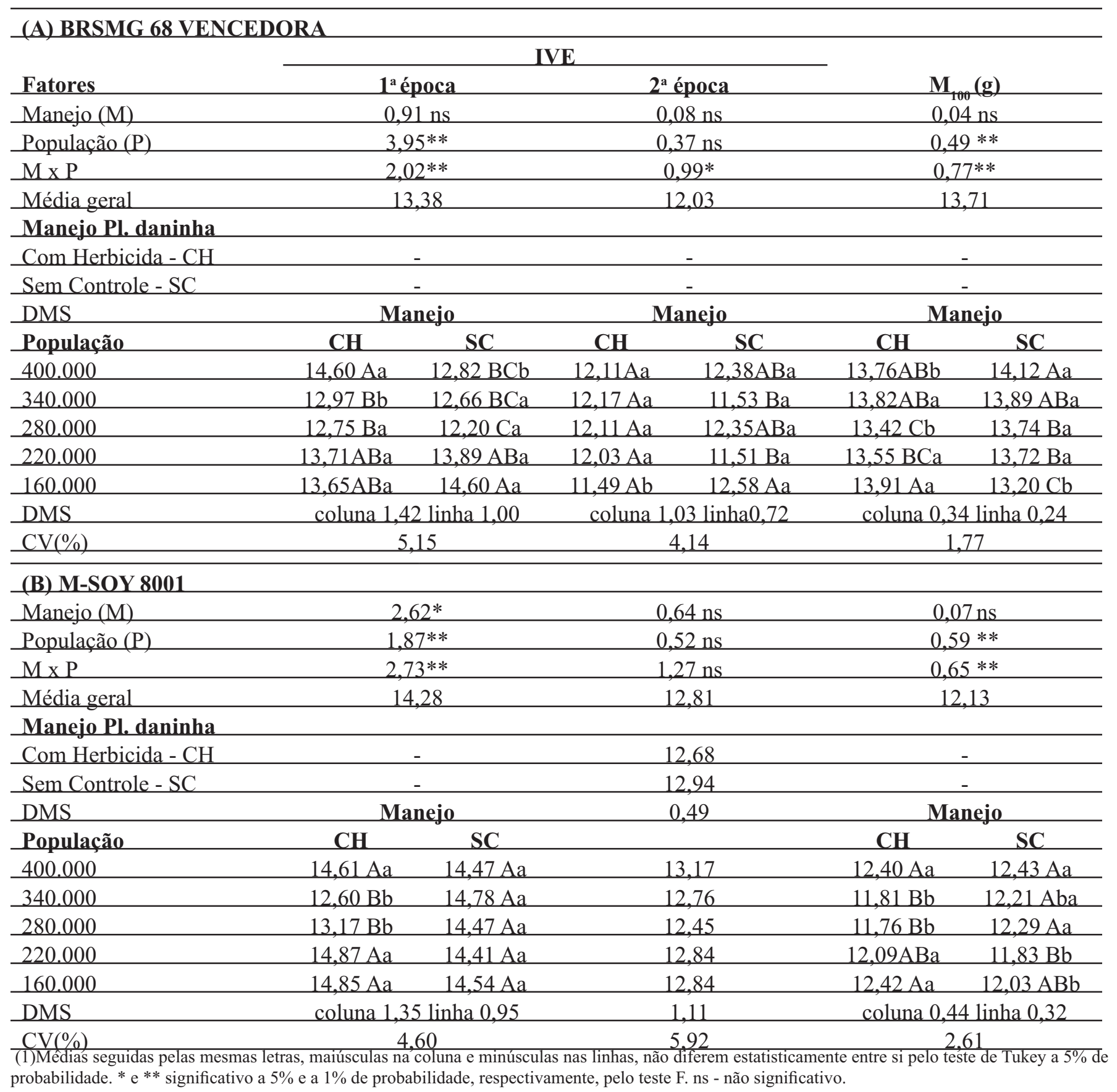


Na Figura 2 estão apresentadas as equações de regressão e os coeficientes de determinação para a $\mathrm{CE}$ em função da população da cv. Vencedora após o armazenamento (2a época). Com a variação da população, observou-se uma tendência inversa entre os resultados provenientes das áreas com herbicidas e os das sem controle de daninhas.

FIGURA 2. Relação entre a condutividade elétrica (CE) (2época) e diferentes populações de plantas da cv. BRSMG 68 Vencedora em áreas com e sem controle de plantas daninhas.

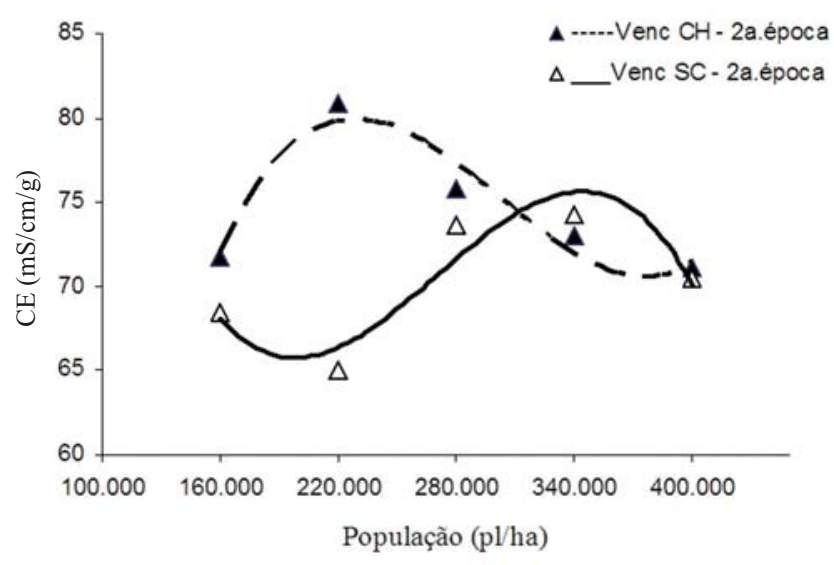

CE $\left(\right.$ Venc $C H-2^{a}$ época $)=5,846.10^{-15} \mathrm{x}^{3}-5,3002 \cdot 10^{-9} \mathrm{x}^{2}+0,00151 \mathrm{x}-$ $57,358 \mathrm{R}^{2}=0,92$

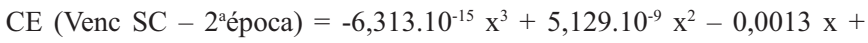
$168,763 \mathrm{R}^{2}=0,87$

$\mathrm{CH}$ - com aplicação de herbicidas e $\mathrm{SC}$ - sem controle de plantas daninhas de probabilidade. $*$ e $* *$ significativo a $5 \%$ e a $1 \%$ de probabilidade, respectivamente, pelo teste F. ns - não significativo.

Já na Figura 3 estão apresentadas as equações de regressão e os coeficientes de determinação em função da população para as cvs. Vencedora ( $1^{\mathrm{a}}$ e $2^{\mathrm{a}}$ épocas) e M-SOY ( $1^{a}$ época) em áreas manejadas com e sem controle de plantas daninhas. Em termos gerais, nota-se uma diminuição do IVE nas populações intermediárias $(340.000,280.000$ e 220.000 pl.ha ${ }^{-1}$ ) havendo uma tendência de igualdade entre a maior e a menor população.

A análise global dos resultados indica uma elevada qualidade fisiológica das sementes de ambas as cultivares, havendo uma aparente pequena superioridade da cv. M-SOY quanto ao vigor (testes de EA e CE na Tabela 2 e IVE na Tabela 3). Os valores da CE obtidos (de 61 a $81 \mu{\mathrm{S} . \mathrm{cm}^{-}}^{-}$ $\left.{ }^{1} . \mathrm{g}^{-1}\right)$ são considerados de alto vigor (Vieira, 1994), embora já apresentem forte tendência a médio vigor (Vieira e Krzyzanowski, 1999).
FIGURA 3. Relação entre o índice de velocidade de emergência (IVE) e diferentes populações de plantas das cvs. BRSMG 68 Vencedora $\left(1^{a} \mathrm{e}\right.$ $2^{\mathrm{a}}$ épocas) e M-SOY 8001 (1 $1^{\mathrm{a}}$ época) em áreas com e sem controle de plantas daninhas.

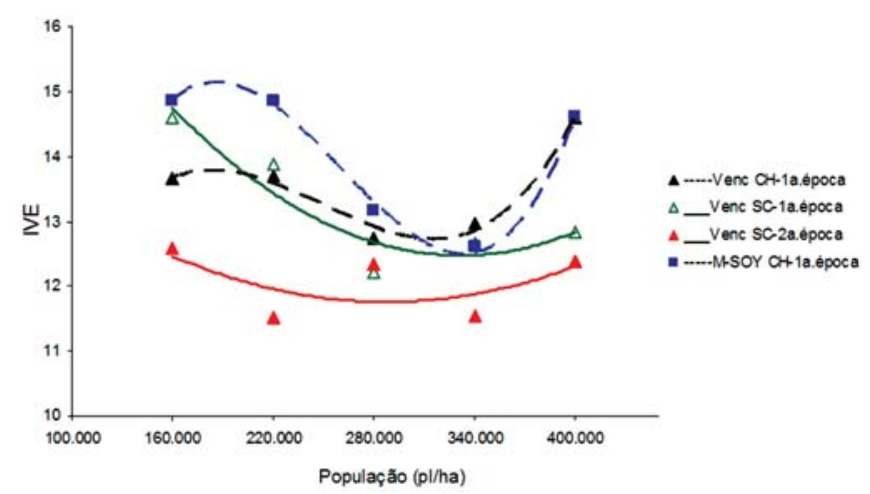

IVE $\left(\right.$ Venc CH $-1^{\mathrm{a}}$ época $)=9,336 \cdot 10^{-16} \mathrm{x}^{3}-6,983 \cdot 10^{-10} \mathrm{x}^{2}+0,000162 \mathrm{x}+$ $1,8125 \mathrm{R}^{2}=0,97$

$\operatorname{IVE}\left(\right.$ Venc SC $-1^{\mathrm{a}}$ época $)=7,733 \cdot 10^{-11} \mathrm{x}^{2}-5,125 \cdot 10^{-5} \mathrm{x}+20,965 \mathrm{R}^{2}=$ 0,87

IVE $\left(\right.$ Venc SC $-2^{\mathrm{a}}$ época $)=4,335 \cdot 10^{-11} \mathrm{x}^{2}-2,4927 \cdot 10^{-5} \mathrm{x}+15,342 \mathrm{R}^{2}=$ 0,34

IVE $\left(\right.$ M-SOY CH $-1^{\text {a }}$ época $)=1,659.10^{-15} \mathrm{x}^{3}-1,292.10^{-9} \mathrm{x}^{2}+0,000308$ $\mathrm{X}-8,1788 \mathrm{R}^{2}=0,99$

$\mathrm{CH}$ - com aplicação de herbicidas e SC - sem controle de plantas daninhas

De modo geral, as condições climáticas favoreceram a obtenção de sementes de soja de boa qualidade. Durante a fase vegetativa, iniciada em 25/11/2002 e finalizada com o início do florescimento $(03 / 01 / 2003)$, a temperatura média e a disponibilidade de chuvas do período, proporcionaram um excedentehídricoadequadoaocrescimento edesenvolvimento das plantas de soja. Na fase reprodutiva, a ocorrência de intensas precipitações proporcionou novamente "superávit", o que garantiu uma adequada granação das vagens de soja. No final do processo de maturação das sementes (fevereiro), a área apresentou um reduzido excedente hídrico $(30 \mathrm{~mm})$ e próximo à maturação de colheita $-\mathrm{R}_{8}$ (março), este se igualou a zero, o que resultou em uma rápida desidratação das sementes, sem a ocorrência de períodos de ganhos e perdas de água pela semente, que poderiam levar a danos, muito embora a temperatura média desse período $\left(24,7{ }^{\circ} \mathrm{C}\right)$ excedesse a ideal de $22{ }^{\circ} \mathrm{C}$ recomendada por Costa et al. (1994).

Diversos trabalhos têm indicado resultados contraditórios quanto à interferência da população sobre a $\mathrm{M}_{100}$. Moore (1991) observou que a massa das sementes aumentava com a diminuição da população, já Tourino et al., (2002) 
encontraram decréscimos e em contrapartida, Crusciol et al., (2002) e Peluzio et al. (2002) relataram não ter verificado variações.

Os resultados dos fatores $\mathrm{M}$ e $\mathrm{P}$ sobre a $\mathrm{M}_{100}$ estão apresentados na Tabela 3 e na Figura 4. De maneira geral, alterações na população de plantas não interferiram na massa de sementes. Na menor população (160.000 pl.ha $\left.{ }^{-1}\right)$, em ambas as cultivares, áreas com herbicidas produziram sementes mais pesadas. Esse desempenho indica que, provavelmente, a baixa população de plantas de soja presente não foi capaz de inibir o crescimento das plantas daninhas (controle cultural), o que fez com que houvesse uma maior competição pelos fatores de crescimento, diminuindo a massa das sementes nas áreas não controladas. Em todas as situações estudadas a cv.
Vencedora superou a M-SOY quanto a $\mathrm{M}_{100}$, o que pode ser explicado pela análise das características genéticas de cada cultivar, onde a Vencedora apresenta uma $\mathrm{M}_{100}$ sementes média de $14,27 \mathrm{~g}$ e a M-SOY de $11,55 \mathrm{~g}$.

O resumo da análise de variância e as médias das porcentagens de retenção nas peneiras 12, 14, 15, 16, 17 e "fundo", encontra-se na Tabela 4. O fator manejo interferiu na porcentagem de retenção de todas as peneiras das duas cultivares, com exceção da peneira 16 da cv. Vencedora e das peneiras 16 e 17 da cv. M-SOY. O "fundo" também não mostrou diferenças determinadas por este fator nas duas cultivares. $\mathrm{O}$ fator população interferiu apenas nas peneiras 15 e 17 da cv. Vencedora. A interação dos fatores M e P foi significativa para a peneira $12 \mathrm{da} \mathrm{cv}$. Vencedora

TABELA4. Análise de variância (quadrado médio) e médias da porcentagem de retenção de sementes nas peneiras $12,14,15,16,17$ e fundo em função do manejo das plantas daninhas e da população de plantas de soja das cultivares BRSMG 68 Vencedora (A) e M-SOY 8001 (B) ${ }^{1}$, Jaboticabal, SP, 2002/03.

\begin{tabular}{|c|c|c|c|c|c|c|}
\hline \multicolumn{7}{|c|}{ (A) BRSMG 68 VENCEDORA } \\
\hline Fatores & P.12 & P.14 & P.15 & P.16 & P.17 & Fundo \\
\hline Maneio - M & $0.84 *$ & $40.60 *$ & $343.98^{*}$ & $400.69 *$ & $33.67 *$ & $0.001 \mathrm{~ns}$ \\
\hline Populacão -P & $0,16 \mathrm{~ns}$ & $11,52 \mathrm{~ns}$ & $190,43^{*}$ & $178,39 \mathrm{~ns}$ & $22.97 * *$ & $0,02 \mathrm{~ns}$ \\
\hline $\mathrm{M} \times \mathrm{P}$ & $0.61 * *$ & $13.77 \mathrm{~ns}$ & $67.68 \mathrm{~ns}$ & $128.39 \mathrm{~ns}$ & $8.64 \mathrm{~ns}$ & $0,01 \mathrm{~ns}$ \\
\hline Média geral & $\frac{0.04}{1.4}$ & 9,6 & 32,0 & 50,0 & 6.5 & 0.5 \\
\hline \multicolumn{7}{|c|}{ Manejo Pl.daninha } \\
\hline $\mathrm{CH}$ & - & $8.6 \mathrm{~B}$ & $29.1 \mathrm{~B}$ & 53.1 & $7.4 \mathrm{~A}$ & 0,45 \\
\hline $\mathrm{SC}$ & - & $10,7 \mathrm{~A}$ & $34.9 \mathrm{~A}$ & 46.8 & $5.5 \mathrm{~B}$ & 0.46 \\
\hline DMS & Maneio & 1.6 & 5.2 & 6.4 & 1.5 & 0,07 \\
\hline \multicolumn{7}{|c|}{$\mathrm{CH} \quad \mathrm{SC}$} \\
\hline 400.000 & $1,1 \mathrm{Aa} \quad 1,2 \mathrm{Ba}$ & 7.5 & $23.5 \mathrm{~B}$ & 57.9 & $9.4 \mathrm{~A}$ & 0.5 \\
\hline 340.000 & $1,6 \mathrm{Aa} \quad 1,3 \mathrm{ABa}$ & 10,5 & $34,1 \mathrm{AB}$ & 47.7 & $5,7 \mathrm{~B}$ & 0,5 \\
\hline 280.000 & $1.4 \mathrm{Aa} \quad 1.4 \mathrm{ABa}$ & 10,2 & $34.2 \mathrm{AB}$ & 48,3 & $5.3 \mathrm{~B}$ & 0,5 \\
\hline 220.000 & $1.8 \mathrm{ABa}$ & 9.8 & $32.6 \mathrm{AB}$ & 50,2 & $5.4 \mathrm{~B}$ & 0.5 \\
\hline 160.000 & $2,1 \mathrm{Aa}$ & 10.1 & $35.6 \mathrm{~A}$ & 45.7 & $6.5 \mathrm{AB}$ & 0.4 \\
\hline DMS & coluna 0,8 linha 0.5 & 3,7 & 11,6 & 14,4 & 3,3 & 0,2 \\
\hline $\mathrm{CV}(\%)$ & 26,50 & 26,2 & 24,88 & 19,70 & 34,96 & 24,32 \\
\hline \multicolumn{7}{|c|}{ (B) M-SOY 8001 } \\
\hline Maneio- M & $110.22 * *$ & $391.88^{* *}$ & $783.22 * *$ & $14.64 \mathrm{~ns}$ & $0.96 \mathrm{~ns}$ & $0.29 \mathrm{~ns}$ \\
\hline Populacão- P & $12.13 \mathrm{~ns}$ & $13,53 \mathrm{~ns}$ & $73,07 \mathrm{~ns}$ & $20.64 \mathrm{~ns}$ & $0,41 \mathrm{~ns}$ & $0,10 \mathrm{~ns}$ \\
\hline $\mathrm{M} \times \mathrm{P}$ & $22,82 \mathrm{~ns}$ & $25,16 \mathrm{~ns}$ & $69,67 \mathrm{~ns}$ & $28,25 \mathrm{~ns}$ & $0,26 \mathrm{~ns}$ & $0,08 \mathrm{~ns}$ \\
\hline Média geral & 11.8 & 28,6 & 47.7 & 9.8 & 1,2 & 0,9 \\
\hline \multicolumn{7}{|c|}{ Maneio Pl. daninha } \\
\hline $\mathrm{CH}$ & $10.1 \mathrm{~B}$ & $25.5 \mathrm{~B}$ & $52.1 \mathrm{~A}$ & 10.4 & 1.0 & 0.9 \\
\hline $\mathrm{SC}$ & $13,5 \mathrm{~A}$ & $31,7 \mathrm{~A}$ & $43,2 \mathrm{~B}$ & 9.2 & 1,3 & 1,0 \\
\hline DMS & 2.6 & 4.3 & 5.4 & 3.0 & 0.6 & 0.2 \\
\hline \multicolumn{7}{|l|}{ Populacão } \\
\hline 400.000 & 11.0 & 27,7 & 48,5 & 10,6 & 1,2 & 1,0 \\
\hline 340.000 & 12,8 & 28.9 & 48.3 & 7.8 & 1,1 & 0.9 \\
\hline 280.000 & 10.2 & 26.8 & $\begin{array}{l}51.0 \\
51.0\end{array}$ & 9.9 & $\frac{1,1}{1.2}$ & 0.8 \\
\hline 220.000 & 118 & 30.0 & 47.7 & 8.8 & 0.8 & 0.8 \\
\hline 160.000 & 13,1 & 29.5 & 42,8 & 12,0 & 1,5 & 1,1 \\
\hline DMS & 5.9 & 9.7 & 12.1 & 6.9 & 1.3 & 0.5 \\
\hline $\mathrm{CV}(\%)$ & 34,40 & 23.13 & $\frac{1 ., 18}{17,38}$ & 47.99 & 77.53 & 39.96 \\
\hline
\end{tabular}

(1)Medias seguidas pelas mesmas letras, maiưsculas na coluna e minúsculas nas linhas, não diferem estatisticamente entre si pelo teste de Tukey a $5 \%$ de probabilidade. $* \mathrm{e} * *$ significativo a $5 \%$ e a $1 \%$ de probabilidade, respectivamente, pelo teste $\mathrm{F}$. ns - não significativo. $\mathrm{CH}-\mathrm{com}$ herbicida e SC - sem controle de plantas daninhas 
FIGURA 4. Relação entre a massa de 100 sementes $\left(M_{100}\right)$ e diferentes populações de plantas das cvs. BRSMG 68 Vencedora e M-SOY 8001 em áreas com e sem o controle de plantas daninhas.

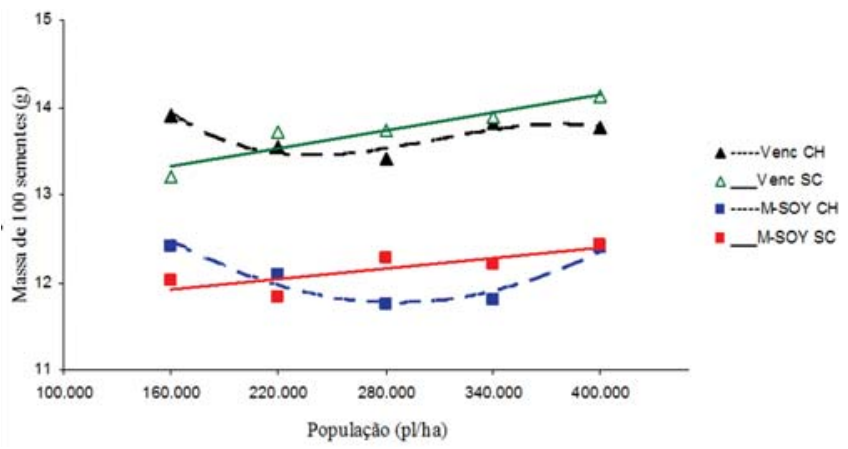

$M_{100}($ Venc $C H)=-2,662 \cdot 10^{-16} x^{3}+2,465 \cdot 10^{-10} x^{2}-7,218 \cdot 10^{-5} x+20,262$ $\mathrm{r}=0,93$

$\mathrm{M}_{100}($ Venc SC $)=0,0000033 \mathrm{x}+12,794 \mathrm{r}=0,94$

$\mathrm{M}_{100}(\mathrm{M}-\mathrm{SOY} \mathrm{CH})=4,442 \cdot 10-^{11} \mathrm{x}^{2}-2,542 \cdot 10-5 \mathrm{x}+15,414 \mathrm{r}=0,96$

$\mathrm{M}_{100}(\mathrm{M}-\mathrm{SOY} \mathrm{SC})=0,0000019 \mathrm{x}+11,602 \mathrm{r}=0,80$

$\mathrm{CH}$ - com aplicação de herbicidas e SC - sem controle de plantas daninhas

A soma das médias gerais da porcentagem de retenção de sementes da cv. Vencedora nas peneiras 15,16 e 17 e das peneiras 12, 14 e 15 da cv. M-SOY, indicam um valor de aproximadamente $88 \%$ (Tabela 4 ). A competição com plantas daninhas levou a uma redução geral no tamanho das sementes em decorrência da menor disponibilidade de luz, nutrientes e água. Braz (1996) relata que, estudos conduzidos em outros países, também mostraram que a presença de plantas daninhas pode influir no tamanho dos grãos produzidos. Em termos gerais não houve interferência da população sobre o tamanho da semente produzida, discordando de Oliveira (2000), que relata reduções no tamanho das sementes com aumentos na densidade de plantas.

\section{CONCLUSÕES}

A cultura da soja é capaz de suportar grandes reduções de população sem perdas estatisticamente significativas de produtividade. Essa capacidade é dependente da cultivar: a BRSMG 68 Vencedora suportou reduções de até $45 \%$ e a M-SOY 8001 de até $30 \%$, em relação à população de 400.000 pl.ha $^{-1}$. Variações na população de plantas de soja não interferem na qualidade fisiológica, no tamanho e na massa das sementes produzidas.

\section{REFERÊNCIAS}

BLANCO, H.G.; OLIVEIRA, D.A.; ARAUJO, J.B.M.; GRAS, N. Observações sobre o período crítico em que as plantas daninhas competem com a soja (Glycine max (L.) Merr.). O Biológico, São Paulo, v. 39, n.2, p.21-35, 1973.

BRASIL. Ministério da Agricultura e Reforma Agrária. Regras para análise de sementes. Brasília, DF: SNDA/ DNDV/CLAV, 1992. 365p.

BRAZ, B.A. Efeitos de redução de distância entrelinhas e de dosagens de latifolicidas no controle de plantas daninhas na cultura de soja [Glycine $\max (\mathrm{L}$.) Merrill]. 1996. 143f. Tese (Doutorado), Universidade Estadual Paulista, Jaboticabal.

COSTA, N.P.; PEREIRA, L.A.G.; FRANÇA-NETO, J.B.; HENNING, A.A.; KRZYZANOWSKI, F.C. Zoneamento Ecológico do Estado do Paraná para produção de sementes de cultivares precoces de soja. Revista Brasileira de Sementes, Brasília, DF, v.16, n.1, p.12-19, 1994.

CRUSCIOL, C.A.C.; LAZARINI, E.; BUZO, C.L.; SÁ, M.E. de. Produção e qualidade fisiológica de sementes de soja avaliadas na semeadura de inverno. Scientia Agricola, Piracicaba, v.59, n.1, p.75-96, 2002.

DENARDI, T.; RAMOS, T.C.; TARTARO, D.E.; NUNES, E. de M.; ASSMANN, I.C.; CICMANEC, E.A. Resposta da cultivar de soja ICA 3 sob cinco densidades de semeadura. In: REUNIÃO DE PESQUISA DE SOJA DA REGIÃO CENTRAL DO BRASIL, 25., 2003, Londrina. Resumos... Londrina: EMBRAPA-CNPSo, 2003. p.241. (EMBRAPACNPSo. Documentos, 209).

ESTAT 2.0. Sistema de análise estatística. Jaboticabal: UNESP - Pólo Computacional - Departamento de Ciências Exatas, 1994.

FEHR, W.R.; CAVINESS, C.E. Stages of soybean development. Ames: Iowa State University of Science and Technology, 1977. 11p.

HICKS, D. R. PENDLETON, J.W.; BERNARD, R.L.; JOHNSTON, T.J. Response of soybean plant types to planting patterns. Agronomy Journal, Madison, v.61, p.290-293, 1969.

KRZYZANOWSKI, F.C.; VIEIRA, R.D.; FRANÇA NETO, J.B. (Ed.). Vigor de sementes: conceitos e testes. Londrina: ABRATES, 1999. 218p.

MAEDA, J.A.; MASCARENHAS, H.A.A.; ALMEIDA, L.D.; NAGAI, V. Influência de cultivares, espaçamentos e localidades na qualidade da semente de soja. Pesquisa Agropecuária Brasileira, Brasília, DF, v.18, n.5, p.515- 
518,1983

MOORE, S.H. Uniformity of planting spacing effect on soybean population parameters. Crop Science, Madison, v.31, n.4, p.1049-1051, 1991.

NAKAGAWA, J.; MACHADO, J.R.; ROSOLEM, C.A. Efeito da densidade de plantas no comportamento de cultivares de soja, em duas épocas de semeadura. Pesquisa Agropecuária Brasileira, Brasília, DF, v.23, n.9, p.10031014, 1988.

OLIVEIRA, B.J. Efeitos da população de plantas no tamanho e na qualidade fisiológica de sementes de híbrido de milho. 2000, 77f. Dissertação (Mestrado) - Universidade Estadual Paulista, Jaboticabal.

PELUZIO, J.M.; BARROS, H.B.; SANTOS, M.M.; REIS, M.S.R. Comportamento de duas cultivares de soja em diferentes populações de plantas, sob condições de várzea irrigada, no sul do Estado do Tocantins. Revista Agricultura Tropical, Cuiabá, v.6, n.1, p.69-80, 2002.

PEREIRA, A.R. Competição intra-específica entre plantas cultivadas. O Agronômico, Campinas, v.41, n.1, p.5-11, 1989.

RAIJ, B. van; CANTARELLA, H.; QUAGGIO, J.A.; FURLANI, A.M.C. Recomendações de adubação e calagem para o Estado de São Paulo. Campinas: IAC, 1997. 285p. (Boletim técnico, 100).
REZENDE, P.M.; VIEIRA, M.G.G.C.; FRAGA, A.C.; FAVORETTO, C.R.S. Efeitos da densidade de plantas sobre a produção, qualidade das sementes e outras características da soja [Glycine max (L.) Merrill]. Revista Ciência e Prática, Lavras, v.9, n.1, p.39-47, 1985.

ROSOLEM, C.A.; SILVÉRIO, J.C.O.; NAKAGAWA, J. Densidade de plantas na cultura da soja. Pesquisa Agropecuária Brasileira, Brasília, DF, v.18, n.9, p.977984, 1983.

TECNOLOGIAS de produção de soja - região central do Brasil 2006. Londrina: Embrapa Soja: Embrapa Cerrados: Embrapa Agropecuária Oeste, 2005. 220 p. (Embrapa Soja. Sistemas de Produção, 9).

TOURINO, M.C.C.; REZENDE, P.M.; SALVADOR, N. Espaçamento, densidade e uniformidade de semeadura na produtividade e características agronômicas da soja. Pesquisa Agropecuária Brasileira, Brasília, DF, v.37, n.8, p.1071-1077, 2002.

VIEIRA, R.D. Teste de condutividade elétrica. In: VIEIRA, R.D.; CARVALHO, N.M. (Ed.). Testes de vigor em sementes. Jaboticabal: FUNEP, 1994. p.103-132.

VIEIRA, R.D.; KRZYZANOWSKI, F.C. Teste de condutividade elétrica. In: KRZYZANOWSKI, F.C; VIEIRA, R.D.; FRANÇA NETO, J.B. (Ed.). Vigor de sementes: conceitos e testes. Londrina: ABRATES, 1999. p. 4.1-4.26. 